punto org

Collana diretta da Luigi Maria Sicca

51 


\title{
IL CANTO DEI SIRENI
}

INVENZIONI TRANS/SINGOLARI E

\author{
PSICOANALISI LACANIANA
}

\author{
Prefazione \\ Antoni Vicens \\ Postfazione \\ Paola Guazzo
}

Editoriale Scientifica

Napoli 
Il volume è stato finanziato da

puntOorg International Research Network

Tutti i diritti sono riservati

(C) 2019 Editoriale Scientifica srl

Via San Biagio dei Librai 39

80138 Napoli

www.editorialescientifica.com

info@editorialescientifica.com

ISBN 978-88-9391-484-0 
Durante un incontro del TGEU, network Transessuale e Transgender europeo,

qualche anno fa, incontrai un@ attivista olandese.

Indossava una gonna al ginocchio e muoveva con eleganza

lunghe gambe pelose, il trucco leggero sul viso si armonizzava

con la leggera peluria sul mento.

Iniziai la sua conoscenza presentandomi, classicamente.

"Mi chiamo Vreer" mi rispose.

"Sai cosa significa in olandese?" mi chiese,

e certo non lo sapevo. Significava "sireno".

Fece una pausa, forse preparandosi a gustare la mia reazione.

"Sireno?" chiesi disorientata. "Sirena si dice, in italiano" la corressi.

"Conosco l'italiano, voglio proprio dire Sireno".

"Ma come? I Sireni non esistono" mi sorpresi a dire di un fiato.

Vreer mi rispose sorridendo: "Neanche le Sirene esistono..."

Rimasi li, stupita e confusa da me stessa.

Andammo poi a bere qualcosa.

A tratti mi sorprendevo a guardare Vreer.

Gli anfibi di pelle stavano così bene con la borsa di stoffa colorata, non avrei mai immaginato di abbinarle.

R.P.*

* Equimesi in Amazzonia, dialoghi intorno alla depatologizzazione della transessualità (2013), a cura di C. Ballarin e R. Padovano, Milano, Mimesis. 
Indice

11 Nota editoriale

di Paolo Valerio

19 Prefazione

di Antoni Vicens

25 INTRODUZIONE

29 1. Freud e l'enigma dei SeSsi

29 1.1. Dal sesso unico al dimorfismo sessuale

32 1.2. Quale libido?

36 1.3. Il complesso di Edipo come operatore psichico

38 1.4. L'angoscia di castrazione e il rifiuto della femminilità per entrambi i sessi

42 1.5. Freud si distanzia dalle teorie dell'epoca rispetto all'inversione sessuale

2. Fili deLL'INSEgNAMENTo di LACAN

2.1. Soggetto dell'inconscio e sessualità

2.1.1. Il soggetto in psicoanalisi

51

54

2.1.2. La novità della metafora paterna e il fallo come operatore di desiderio

2.1.3. Mi dice questo ma cosa vuole? Oscillazione tra alienazione e separazione

57 2.2. Dalla dialettica del desiderio all'irriducibilità del godimento

60

2.2.1. Una clinica orientata dal Reale e il nodo borro meo come scrittura della clinica

61 2.2.2. Dal soggetto dell'inconscio al parlessere, il traumatismo della lalingua 
64

66

71

71

73

74

74

75

76

78

80

82

86

88

88

89

90

95

95

100

102

103

107 5. TRANS/INVENZIONI

107 non-tutto

3.1. Il terzo sesso

3.2. Discorsi scientifici biologiche za di genere alcuni altri

2.2.3. Non c'è rapporto sessuale

2.2.4. Sessuazione, il godimento fallico e il godimento

3. DisCORSI INTORNO ALLA QUESTIONE TRANS

3.2.1. Magnus Hirschfeld e il neologismo travestito

3.2.2. Havelock Ellis: dall'inversione sessuale estetica all'eonismo

3.2.3. $D a$ kontrare Sexualemfindung $a$ sselischer Transsexualismus

3.2.4. Henry Harry Benjamin: nasce il significante transessualismo

3.2.5. J. Robert Stoller e la differenza tra i concetti di genere e sesso

3.2.6. John William Money e lo studio delle varianti

3.2.7. Il dibattito natura/cultura

3.2.8. Dal disturbo dell'identità di genere alla disforia dell'identità di genere

3.3. Femminismi, attivismo LGBTQI e teoria queer

3.3.1. I femminismi

3.3.2. L'attivismo LGBTQI

3.3.3. Le teorie queer e la psicoanalisi lacaniana

4. LA PSICOANALISI LACANIANA E LA QUESTIONE TRANS

4.1. Una questione di godimento al di là della sembian-

4.2. L'essere sessuato non si autorizza che da sé e con

4.3. Una clinica della contingenza

4.4. La bussola di ogni psicoanalista

5.1. L'impasse nell'incontro con l'Altro sesso 
108 5.2. Scacco matto

$110 \quad$ 5.2.1. Essere scelto

111 5.2.2. Una vita da uomo

112 5.2.3. Un segreto

115 6. TRANS/GENERI

115 6.1. Nuovi modi di nominarsi

117 6.2. Mat*Ang* l'equilibrista

119 6.2.1.Non ne posso più di vivere così

121 6.2.2. Scollegato, io fra di voi

122 6.2.3. Matteo

123 6.2.4. Angelica, una via singolare verso la femminilità

124 6.2.5. Matt*Ang

127 7. Al DI LÀ DELLA SEMBIANZA DI GENERE

127 7.1. Quale godimento?

128 7.2. Rebecca, Non-tutta, non per tutti

130 7.2.1. Figlio degenere

130 7.2.2. Donna trans

131 7.2.3. Rebecca, non con tutti

132 7.2.4. Essere scelta da un uomo

133 7.2.5. Un nuovo amore, la contingenza del reale si presentifica

135 8. Nel TEMPo DELLA GENitorialità

135 8.1. Madri - Padri o Genitori?

137 8.2. Trans/ma (pa) ternità

141 9. Conclusioni

141 9.1.Dalla logica dell'identificazione all'irriducibile del godimento

145 Intervento

Christian Ballarin 
149 Postfazione

Paola Guazzo

153 Bibliografia

165 Indice dei nomi

167 Hanno scritto nella Collana punto org 


\section{Nota editoriale}

Paolo Valerio*

Con la pubblicazione di quest'opera di Mary Nicotra diamo seguito a una linea di ricerca che connota da alcuni anni l'impegno di puntOorg International Research Network intorno (e interna) al tema dell'inclusione nelle sue plurali articolazioni.

In linea con il lavoro condotto in seno al Centro Servizi per l'Inclusione Attiva e Partecipata degli Studenti (SInAPSi) dell'Università degli Studi di Napoli "Federico II", per noi inclusione:

assume un significato ben diverso dalla logica della integrazione, che immette chi è fuori, all'interno di cornici che vengono lasciate però invariate. L'inclusione, insomma, per noi è riorganizzazione: attivata da una richiesta di chi è fuori, imponendo a chi è dentro di rivedere - insieme con chi (era) inizialmente fuori - vocabolari teorici, procedure, istituzioni, in modo da rimuovere le cause dell'esclusione (Sicca, 2018).

Lanciammo la questione (Oliverio, Sicca, Valerio, 2015) con una ricerca che metteva in dialogo lo sguardo degli studi di Organizzazione aziendale e di gestione delle Risorse umane, quello psicologico e quello pedagogico, arricchito da testimonianze di vita vissuta nel mondo delle

* Professore ordinario di Psicologia clinica, Università degli Studi di Napoli “Federico II". 
organizzazioni. Quella fu anche un'occasione per attivare e istituzionalizzare la partnership internazionale tra SInAPSi, il Gender \& Equality Office dell'University of Essex e la Fondazione Genere Identità Cultura.

Due ulteriori collaborazioni (Boncori, 2017; 2018; Bizjak, 2018) ampliarono la sfera della nostra rete, sia perché riferiti a contesti accademici di area anglosassone sia in termini di riflessione teorica sulla dialettica tra inclusione ed esclusione.

Ad alimentare il dibattito, ci fu una ulteriore nostra "risposta": se nella pubblicazione del 2015 l'inclusione era filtrata attraverso il cristallino delle pratiche di lavoro, successivamente ci dedicammo a comprendere le dinamiche organizzative che interessano:

chi, da quel mondo, è fuori, perché "dentro" : grazie al Protocollo d'Intesa "Al di là del muro" siglato inizialmente tra il Centro SInAP$\mathrm{Si}$, la Fondazione Genere Identità Cultura (G.I.C.) e la Casa Circondariale Poggioreale, affrontammo la questione su come favorire e promuovere un sostegno professionale specifico agli Operatori Penitenziari (funzionari giuridico pedagogici, poliziotti penitenziaria, Psicologi, Dirigenti Responsabili) dei Reparti "Salerno" e "Roma" (in quanto ospitanti detenuti omosex e transex della C.C.), impegnati nelle fasi critiche dell'accoglimento e del trattamento di reclusione dei detenuti omosessuali e transessuali (Sicca, 2018).

$* * *$

Seppure cursoriamente, vorrei evidenziare come le questioni qua evocate trovino sponda in ulteriori aree di ricerca puntOorg, solo apparentemente distanti da quelle richiamate, ma di fatto assai contigue nell'ottica di una inclusività epistemologica prima ancora che (esclusivamente) fattuale: mi riferisco alle ricerche relative al mondo della formazione e della filosofia dell'educazione che rappresentano una sfida di lungo periodo rispetto alla dimensione etica 
della questione su come-si-fa, che è tecnica (téchne, $\tau \dot{\varepsilon} \chi v \eta)$, che nel mondo antico è arte, quindi percezione del mondo, dei mondi, da cui artefatti, artis e factum, fatto ad arte, effetto dei sensi che danno senso: mi riferisco per esempio al lavoro di Francesco Piro (2016), da un lato, ma anche il più recente percorso di analisi delle "Sfide didattiche. Il pensiero critico nella scuola e nell'università" (Piro, Sicca et al., 2018), dove oltre alla riflessione sulle cosiddette "discipline tradizionali", la sfida dell'educazione include anche la riflessione sulla musica, area di ricerca privilegiata in puntOorg, anche perché considerata potente fonte di conoscenza manageriale (Sicca, 2012), per un costante rinnovamento nelle scienze umane e sociali (Diana, Sicca e Turaccio, 2017).

Entro questa fucina, la cui articolazione e complessità è stata sinteticamente proposta, si innesta il lavoro di Mary Nicotra: l'insufficienza della visione binaria "femminile-maschile" o "donna- uomo" dà ragione di quanto sia delicato, da maneggiare con cura, l'intreccio tra corpo e desiderio, facendo i conti con un sistema simbolico che richiede nuovi vocabolari teorici e un modo di produrre senso alternativo alla (e al tempo stesso rispettoso della) logica delle scienze che, forti dei successi e delle rassicurazioni proprie del metodo riduzionistico di laboratorio, rischiano di perdere pezzi di conoscenza.

La psicoanalisi lacaniana soccorre a questa questione di fondo, proprio a partire dai temi trattati nel lavoro di Mary Nicotra, che attraverso la pratica clinica, evidenzia come l'assegnazione biologica maschio/femmina non perfeziona, una volta per tutte, la questione dell'identità.

Il testo di Nicotra affronta la questione trans*, in tutte le sue declinazioni (è questa la ragione del ${ }^{*}$, gesto plurale), prendendo le mosse da due autori: Freud e so- 
prattutto Lacan. Senza soffermarci su tutti gli aspetti che 1'Autrice sviluppa dei due psicoanalisti, diremo semplicemente la ragione di questa scelta: nell'essere umano la psicosessualità, se possiamo usare questo termine, non è una variabile dipendente del sesso biologico. Benché le tesi di Freud sulla fase fallica e sul carattere normativizzante dell'Edipo, così come la frase freudiana secondo cui "l'anatomia è il destino" possano dare adito a degli equivoci, va subito detto che l'Autrice evidenzia che per il padre della Psicoanalisi il legame tra la pulsione sessuale e l'oggetto non è per nulla stretto, il che significa che già Freud aveva aperto una strada per pensare la dimensione gender, diremmo noi, ossia una dimensione non vincolata alla scelta oggettuale, dato che quest'ultima si presenta come contingente. Ed è qui che Lacan permette a Nicotra di compiere un passo più in là, soprattutto grazie alla nozione di godimento.

Lasciando da parte le scansioni che Nicotra individua, sulla scia di Miller, nell'insegnamento di Lacan in riferimento all'assunzione del proprio sesso - dall'inconscio strutturato come un linguaggio, alla centralità del significante fallo e alla correlata funzione del Nome del Padre, con l'articolazione dell'Edipo freudiano in tre tempi fino alla dialettica dell'alienazione e della separazione - andremo direttamente al cuore della questione che qui ci interessa, ossia quella del godimento. Tale questione si articola in Lacan con la tesi che non solo il soggetto è diviso, ma lo è anche l'Altro. Dire che 1'Altro del linguaggio è diviso, ossia mancante, significa questo: io non troverò mai nell' Altro qualcosa, un significante per esempio, che possa rispondere della mia identità, cioè dire io chi sono. Questo vuol dire che l'essere umano non può trovare nessuna garanzia nell'Altro, e per essere precisi niente che gli garantisce che il suo godimento è giusto o sbagliato, 
perché non c'è nessun garante. Se qualcuno pretende di detenere la risposta alla nostra domanda di identità, ossia pretende di essere un legislatore in grado di rispondere a questa domanda, ebbene possiamo essere certi che si tratta di un impostore. Come scrive l'Autrice, riprendendo questa tesi radicale di Lacan:

C'è qualcosa che sfugge alla significazione, un impossibile a dirsi che riguarda il reale che per ogni soggetto si produce appunto come l'impossibile con cui si trova a fare nella propria vita" (p. 57 corsivo mio).

Sottolineiamo il termine, perché è proprio il godimento. Lacan arriva a dire che tutto ciò che resiste alla presa del Simbolico nella realtà può essere chiamato godimento, godimento che è articolato anche se non articolabile, il che significa che ha una sua posizione logica nell'esperienza del Soggetto (ivi).

A questo punto, si comprende perché Nicotra affronta la questione trans ${ }^{*}$. Se siamo prigionieri dei dispositivi discorsivi e sociali (si pensi all'uso che Nicotra fa di Foucault), allora «nella nostra epoca è importante rimettere in gioco i modi di godimento» (p. 96), e questo è reso possibile proprio da tale questione. Mettendo in evidenza il limite della lettura che Butler propone di Lacan, l'Autrice evidenzia invece il carattere fecondo delle tesi dello psicoanalista francese. Notiamo soltanto di sfuggita che nella pratica lacaniana, l'analista non elargisce nessun sapere, e non può farlo perché nell' Altro manca quel significante che garantirebbe la possibilità di rispondere in modo normativo alla questione dell'identità del soggetto e alla sua modalità di godimento.

Discutendo il connubio transessualità-psicosi, l'Autrice scrive qualcosa di molto importante, che ci porta al di là della questione della patologia. 
Come la clinica insegna può succedere che per un soggetto la transessualità possa diventare il sinthomo, che svolge funzione di annodamento dei tre registri Reale, Simbolico, Immaginario e con l'ultimo Lacan nella clinica non è più la diagnosi a fare da bussola per gli psicoanalisti, ma piuttosto il reale e il godimento in gioco in ogni cura (p. 97).

Questo passaggio è a nostro avviso decisivo. Che cosa vuol dire? Teniamo conto che il termine sinthomo ha un'accezione particolare nella topologia borromea di Lacan, e per limitarci a ciò che ci interessa, diciamo semplicemente che è ciò che permette a un soggetto di annodare il Simbolico, l'Immaginario e il Reale, e dunque che permette a questo soggetto di tenere. Allora, affermare che la transessualità può diventare sinthomo, equivale a dire che un soggetto può trovare nella transessualità la possibilità di annodare i tre registri e, da qui, trovare una propria consistenza. Di conseguenza, il godimento transessuale assume il valore di anello che permette di tenere. Questo vuol dire che non c'è un sapere normativo sulla sessuazione, il che permette all' Autrice di trarne questa conclusione:

Così per ognuno, la questione della sessuazione porta con sé una ricerca intorno al doversi arrangiare rispetto all'essere donna o all'essere uomo, che è sempre una questione complicata, intima, enigmatica e non generalizzabile; ciò riguarda tutti: cisgender, transgender, transessuali (p. 101).

$$
* * *
$$

Mi piace concludere questa nota editoriale annunciando come il felice cortocircuito tra istituzioni accademiche, associazionismo di ricerca e movimenti, ha recentemente visto un protocollo di intesa alla presenza del Rettore dell'Università degli Studi di Napoli "Federico II" e dell'Ambasciatore di Cuba in Italia tra il Centro SInAPSi 
dell' Ateneo federiciano e il Cenesex (Centro Nazionale per l'Educazione Sessuale, Istituto cubano di insegnamento, ricerca e assistenza nel campo della sessualità) diretto dalla dott.ssa Mariela Castro. Ciò con l'intenzione non solo di avviare uno scambio teso a incentivare la promozione di una cultura che valorizzi le differenze e per contrastare le discriminazioni associate al genere e allo stigma sessuale, ma anche con l'intento di testimoniare come e quanto sia possibile riannodare il doppio filo di un sguardo sul mondo (quello proprio della tradizione industriale dell'economia di mercato e quello della esperienza cubana dal secondo Dopoguerra), facendo proprio leva su quella idea di "inclusione" evocata in apertura, nella misura in cui i "margini" sanno (anche a fatica) fecondare il "centro": una leva in grado di dare la rotta verso traguardi ulteriori, spostando insomma sistematicamente in avanti, inesorabilmente, la frontiera del lavoro di ricerca accademica - e un'azione - sempre appena più in là. Senza possibilità di sostare, senza mai giungere a una meta finita.

Paolo Valerio Napoli, Università degli Studi Federico II 